\title{
Jerarquización de competencias emocionales a través del modelo de la pirámide invertida
}

\author{
De José Belzunce, Michael* \\ Danvila del Valle, Ignacio** \\ Martínez-López, Francisco J.*** \\ Sastre Castillo, Miguel Ángel ${ }^{* * * *}$
}

\section{Resumen}

Las teorías de la inteligencia emocional tratan de identificar las competencias emocionales más significativas de cara al éxito personal y profesional, sin embargo, no se centran tanto en establecer niveles de jerarquía entre las mismas. En tal sentido, el objetivo de la investigación es realizar un estudio exploratorio sobre las competencias emocionales con el fin de aportar un modelo: el de la pirámide invertida, que permita su relación y jerarquización para poder predecir el nivel de desarrollo de algunas a partir de los niveles de otras. Para ello se realiza un Panel Delphi con 16 directivos de las grandes compañías que operan en la bolsa de Madrid (IBEX 35). Los resultados obtenidos muestran una relación jerárquica de las competencias emocionales de los altos directivos de las grandes empresas a partir de su categorización en subyacentes, básicas y ejecutivas. El estudio realizado destaca que las competencias más valoradas en las empresas son la escucha activa y la comunicación efectiva. Del modelo propuesto se infiere que para desarrollar estas competencias hay que trabajar

Recibido: 21-11-12. Aceptado: 12-11-12

* Doctor en Administración y Dirección de Empresas, Directivo de empresa, Profesor de International MBA en ESCP Europe y ESIC Business School, e-mail: michaeldejose@gmail / masastre@ emp.ucm.es

** Doctor en Administración y Dirección de Empresas, Profesor Contratado Doctor, adscrito al Departamento de Organización de Empresas de la Universidad Complutense de Madrid, e-mail: idanvila@ccee.ucm.es

*** Doctor en Administración y Dirección de Empresas, Profesor Titular, adscrito al Departamento de Organización de Empresas de la Universidad de Granada, Miembro del Grupo de Marketing del Departamento. de Economía y Empresa de la Universitat Oberta de Catalunya, e-mail: fjmlopez@ugr.es

**** Doctor en Administración y Dirección de Empresas, Profesor Catedrático de E.U., adscrito al Departamento de Organización de Empresas de la Universidad Complutense de Madrid. Profesor Visitante del Instituto Tecnológico Autónomo de México (ITAM), e-mail: masastre@ emp.ucm.es 
Jerarquización de competencias emocionales a través del modelo de la pirámide... De José Belzunce, M.; Danvila del Valle, I.; Martínez-López, F. y Sastre Castillo, M.

sobre varias competencias básicas clave, como son la iniciativa, la flexibilidad, el optimismo y la empatía, características que parecen configurarse como esenciales para trabajar en un contexto de crisis como el actual.

Palabras clave: Inteligencia emocional, competencias emocionales, pirámide invertida, habilidades directivas.

\title{
Hierarchical Structuring of Emotional Competences through the Inverted Pyramid Model
}

\begin{abstract}
Emotional intelligence theories try to identify the most significant emotional competences related to personal and professional success; however, they do not focus much on establishing hierarchical levels among them. The objective of this research is to conduct an exploratory study about emotional competences and offer an inverted pyramid model that permits their interrelation and hierarchical structuring, in order to predict the level of development for some based on the levels of others. To accomplish this, a Delphi Panel was constituted with 16 directors from large companies that operate on the Madrid stock market (IBEX 35). The results show a hierarchical relationship for the emotional competences of top directors in large companies based on their categorization into underlying, basic and executive competences. The study emphasizes that the most valued competences are active listening and effective communication. Using the proposed model, it can be inferred that, to develop these competences, various basic key competences must be worked on, such as initiative, flexibility, optimism and empathy, characteristics that seem essential for working in a context of crisis such as the present one.
\end{abstract}

Key words: Emotional intelligence, emotional competences, inverted pyramid, directorial abilities.

\section{Introducción}

En las últimas décadas, pocos conceptos han suscitado tanto interés simultáneamente en los ámbitos académico, empresarial y en la población en general, como el de la inteligencia emocional (IE). Quizás la razón estribe en que la aplicación del concepto trasciende el mundo de la empresa, ya que es fácil encontrar aplicaciones en la vida personal y familiar; además, la divulgación de algunos modelos a través de libros, que a la postre han resultado best sellers, también ha contri- buido a que numerosas personas se hayan sentido atraídas hacia el concepto de IE.

No obstante, no existe un acuerdo absoluto acerca de las competencias que lo conforman. Algunas teorías se han ido imponiendo en la literatura especializada, pero aún así, la discusión acerca de qué es la IE permanece vigente. Al respecto, se realiza un repaso de las principales visiones acerca de la IE, fundamentado en autores como, Danvila y Sastre (2010); Gardner (1983); Salovey y Mayer (1990); Bar-On (1997); Goleman (1995); Boyat- 
zis (1982); Howard y Bray (1988), entre otros, todo ello, sirve de base para la construcción de una propuesta de competencias definidas por niveles, cuya utilidad es proponer cómo una determinada competencia emocional puede ser desarrollada a partir de aquellas otras con las que está relacionada.

Se propone un modelo denominado pirámide invertida de las competencias emocionales (PICE), el cual se trata de validar a través de la opinión de un conjunto de directivos de grandes empresas españolas. La metodología empleada para este fin ha sido la constitución de un panel Delphi, que ha permitido jerarquizar las competencias $\mathrm{y}$, a partir de las relaciones verticales definidas por el modelo, se ha procedido a establecer un mapa de competencias de los directivos, clave para cualquier organización. Para la aplicación de las encuestas se utilizó una herramienta de apoyo, sustentada en un sistema vía Internet, con una presentación previa de los objetivos e instrucciones para cumplimentar las encuestas, y una clarificación de los conceptos relativos a la IE y a las competencias emocionales.

\section{Inteligencia emocional: Un análisis teórico}

Según Danvila y Sastre (2010), el término IE se utilizó de manera incidental en la crítica literaria en los años sesenta (Van Ghent, 1961) y en la psiquiatría (Leuner, 1966), y en los años ochenta, pasó a emplearse de una manera más sistematizada (Payne, 1986).

Gardner (1983) incluyó la "inteligencia personal" en su obra fundamental en la teoría de las inteligencias múltiples.
Según este autor, la inteligencia personal estaba compuesta por la inteligencia intrapersonal, referida al conocimiento de los aspectos internos de una persona, y por la inteligencia interpersonal, basada en la capacidad básica para percibir diferencias personales en otros individuos, en particular, el contraste en sus estados de ánimo, temperamentos, motivaciones e intenciones (Gardner, 1993).

Salovey y Mayer (1990), en los años noventa, definieron explícitamente la IE, desarrollando una teoría y estableciendo criterios para su medición. Dichos autores definieron la IE como un subconjunto de la inteligencia social que implica la capacidad para controlar los sentimientos y emociones propios y de los demás, para discriminar entre ellos y utilizar esta información para guiar el pensamiento y las acciones.

Estos mismos autores actualizaron años más tarde el concepto, estableciendo una definición más completa. Según ésta, la IE sería "la capacidad de percibir con exactitud, valorar y expresar emociones; la capacidad de encontrar y/o generar sentimientos cuando éstos faciliten el pensamiento y la capacidad de comprender y regular las emociones para promover el crecimiento emocional e intelectual" (Mayer y Salovey, 1997:5). Este modelo conceptual propuesto por Mayer y Salovey (1997) y Mayer, Caruso y Salovey (1999) es el que ha generado mayor número de investigaciones, por las siguientes razones: dispone de una base teórica sólida, contiene una novedosa forma de medirla y ha sido ratificado por numerosos estudios prácticos en múltiples campos (educación, medicina, entre otros). 
Jerarquización de competencias emocionales a través del modelo de la pirámide... De José Belzunce, M.; Danvila del Valle, I.; Martínez-López, F. y Sastre Castillo, M.

Bar-On (1997) consideró un concepto de IE más amplio que el de Salovey y Mayer (1997). Incluyó la intersección de competencias, habilidades y facilitadores que contribuyen a entender y expresarnos, entender a otros y relacionarnos con ellos. También puso el énfasis en los aspectos no cognitivos, con lo cual se distanciaba de otros conceptos de inteligencia.

Para Bar-On (1997), la IE comprende cinco factores de alto nivel: habilidades intrapersonales, habilidades interpersonales, capacidad de adaptar nuestras emociones, capacidad de gestionar el stress y controlar las emociones y capacidad de tener emociones positivas.

Goleman (1995) consideró cinco elementos clave en la IE: conocimiento de las propias emociones, gestión de las emociones, automotivación, reconocimiento de las emociones de los demás y gestión de las relaciones con los demás. Este autor definió en cambio la IE por exclusión: como cualquier característica deseable de carácter personal que no está representada por la inteligencia cognitiva, estableciendo por tanto, dos categorías de inteligencia personal.

Las definiciones dadas, tanto por Goleman (1995) en su trabajo original y en las reformulaciones posteriores de su modelo (Goleman, 2001; Enmerling y Goleman, 2003), como por Mayer y Salovey (1997), pueden considerarse complementarias; de hecho, el creciente auge adquirido por este concepto se ha debido en gran parte a que el conocimiento y las emociones se interrelacionan, y explican los niveles de éxito en diversos ámbitos de la vida. Por tanto, para numerosos autores, el conocimiento o la inteligencia cognitiva no se puede considerar como el único predictor de éxito (Dulewicz y Higgs, 2000; Cherniss, 2001; Mandell y Pherwani, 2003).

Investigaciones empíricas llevadas a cabo por Boyatzis (1982) y Howard y Bray (1988), han mostrado que la posibilidad de predecir el rendimiento de un dirigente depende de un conjunto de competencias que pueden ser agrupadas en tres categorías (Boyatzis y Saatcioglu, 2008): 1) Competencias de inteligencia cognitiva, como los sistemas de pensamiento, 2) Competencias de inteligencia emocional o habilidades intrapersonales, tales como la capacidad de adaptación, 3) Competencias de inteligencia social o habilidades interpersonales, como las redes.

Además de los conocimientos y competencias, el elemento necesario para un rendimiento superior, parece ser el deseo de utilizar el propio talento. Esto viene dado por los valores de una persona, tales como su filosofía, los motivos inconscientes y los rasgos (Boyatzis y Sala, 2004; Boyatzis, 2006). Estos tres dominios de la capacidad o talento (los conocimientos, las competencias y los controladores de tipo motivacional), nos facilitan la comprensión de lo que una persona puede hacer (conocimiento), el modo en el que puede hacerlo (competencias), y los motivos por los que los hace (valores, motivaciones y disposiciones inconscientes).

Las habilidades intrapersonales e interpersonales, que definen la IE, pasan a ser claros predictores de éxito en todos los sentidos. De hecho, como señalan Jamali et al. (2008), la investigación muestra que las personas con más altos niveles de IE alcanzan un mayor éxito en la vida (Bar-On, 1997), mayor éxito profesional (Dulewicz y Higgs, 1998; Weisin- 
ger, 1998), realizan su trabajo con mayor eficacia (Higgs y Rowland, 2002; Prati et al., 2003), se adaptan mejor a las situaciones estresantes (Nikolaou y Tsaousis, 2002) y afrontan mejor el desarrollo de sus planes e ideas (Bar-On, 2000).

Asimismo, según Koman y Wolf (2008), los que tienen niveles superiores de IE incrementan la eficacia en el trabajo en equipo (George, 2000; George and Bettenhausen, 1990; George, 1995; Gardner and Stough, 2002).

Analizando el éxito profesional, los predictores no cognitivos han ido cobrando fuerza en la psicología industrial para procesos de selección, debido a los problemas existentes en las pruebas cognitivas para predecir el rendimiento en el trabajo (Schmidt y Hunter, 1998).

A pesar de la defensa que los principales autores de esta corriente realizan de la aplicación práctica de su teoría, y de su impacto en la eficiencia de las organizaciones (Goleman, 1998; Goleman, Boyatzis y McKee, 2002a, 2002b y 2004), los medios de difusión podrían haber exagerado su validez (Fernández-Berrocal y Extremera, 2006), y en consecuencia, es deseable una mayor profundización en su conceptualización y un mayor esfuerzo para su contrastación empírica. Por ello, en este trabajo se propone un modelo de relación entre las competencias emocionales a partir de una jerarquización de las mismas.

\section{Competencias emocionales}

Para desarrollar las funciones típicas de la función directiva, el directivo-líder deberá poseer y ejercitar una serie de competencias, tanto técnicas (dirigidas más a la función cognitiva del cerebro), como emocionales (orientadas a la función emocional del cerebro). Hasta hace poco, el mundo empresarial sólo reconocía la necesidad de desarrollar las competencias técnicas: visión estratégica, conocimiento del negocio, entre otras; en consecuencia, se ponían los medios para su desarrollo, como si sólo tuvieran un componente cognitivo. No obstante, los resultados de este enfoque limitado han sido parciales, con una gran variación en la eficacia de la misma, y sobre todo en su capacidad para realmente cambiar el comportamiento del directivo.

Cuando Goleman (1995) da a conocer al público en general, y a la comunidad empresarial en especial, el concepto y el potencial de la IE, se produce una revolución (aún en curso) en la comprensión de los factores que explican el éxito y el fracaso de los directivos, así como en el papel de las emociones en la dirección de las personas. Numerosas investigaciones, artículos y libros intentan profundizar y desarrollar este concepto. Inevitablemente, en este proceso se genera una confusión en el ámbito, produciéndose el fenómeno de la extensión del término (aplicación poco rigurosa a demasiados campos). Además, tiene lugar un falso dualismo, diferenciando radicalmente las competencias emocionales de las habilidades directivas; o una mezcla indiscriminada entre conceptos psicológicos, empresariales, médicos y de espiritualidad, que hacen casi imposible entender el sentido de una competencia directiva.

Se pretende en este estudio, delimitar y aclarar los conceptos relacionados con las competencias directivas, proponiendo una clasificación diferente de 
Jerarquización de competencias emocionales a través del modelo de la pirámide... De José Belzunce, M.; Danvila del Valle, I.; Martínez-López, F. y Sastre Castillo, M.

las mismas. Asimismo, se pone de manifiesto lo que aporta la IE al quehacer tradicional del directivo, y a las herramientas de gestión que siempre ha manejado.

Se destaca que en el mundo empresarial, la gran mayoría de las competencias directivas tienen dos componentes: una técnica y otra emocional. Es necesario identificar cuáles son, y completar las características de las competencias técnicas, con el aspecto emocional que conlleva su ejercicio práctico. La hipótesis propuesta es que estas competencias directivas, técnico-emocionales, coinciden con el concepto tradicional de habilidades o competencias directivas. Por otro lado, hay otras competencias directivas cuyo contenido es mayoritariamente emocional, y que deben ser definidas, desarrolladas y tratadas como tales. Este modelo propone una lista de las mismas, y las desarrolla mediante una nueva clasificación en competencias emocionales subyacentes y básicas, consideradas unitariamente en la Figura 1; la definición de las funciones directivas que incorporamos al modelo es la propuesta (y universalmente aceptada) de Koontz y O’Donnell (1964).

Un rasgo esencial del modelo es la nueva propuesta de clasificación de las competencias emocionales, en la que se distinguen tres competencias emocionales diferentes: subyacentes, básicas y directivas o ejecutivas:

1. Las competencias emocionales subyacentes (autoestima, autoconciencia, autodominio y la percepción de otros) pertenecen más al ámbito personal y psicológico del directivo, y constituyen una parte fundamental de su forma de ser y actuar. Este tipo de competencias influyen prácticamente en todo el resto de las

Figura 1

Mapa conceptual de competencias técnicas y emocionales

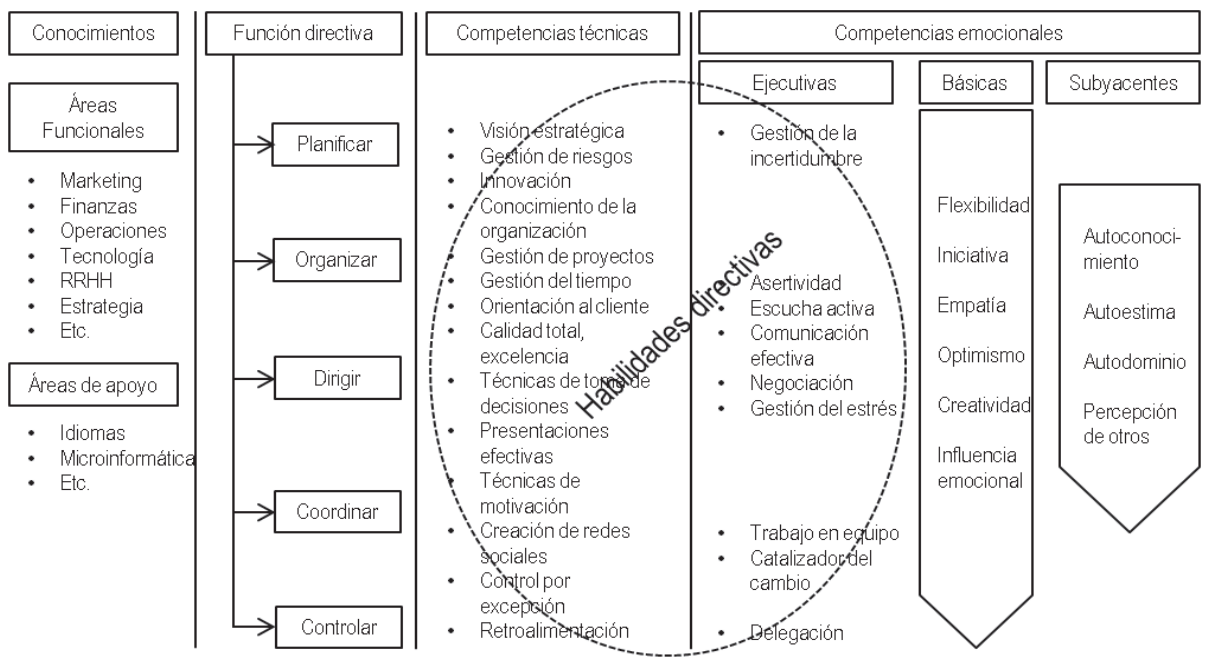

Fuente: Elaboración propia. 
competencias emocionales, aunque en distinta intensidad y grado:

- Tienen una influencia directa sobre las competencias emocionales básicas, como la iniciativa, la empatía o la flexibilidad.

- Su influencia es indirecta, es decir, a través de las competencias emocionales básicas, sobre un nivel superior de competencias que denominaremos competencias ejecutivas (como la negociación o el trabajo en equipo).

- A mayor desarrollo y dominio de las competencias emocionales subyacentes, mayor facilidad tiene el directivo líder en capacitarse en el resto de las competencias directivas.

Sintetizando se podría decir, que las competencias subyacentes están directamente vinculadas al autoconcepto de la persona, siendo difícilmente desarrollables a través de acciones formativas.

2. Las competencias emocionales básicas se pueden dar en cualquier puesto y función, y todo directivo debe aspirar a tener un buen nivel en estas competencias. Sin ellas, difícilmente puede desarrollarse de forma armoniosa competencias emocionales más sofisticadas o complejas.

En esta categoría, por tanto, se recogen aquellas competencias emocionales referenciadas en modelos clásicos que son tan aplicables al mundo de los negocios como al resto de aspectos generales del comportamiento de la persona, es el caso de la flexibilidad, la iniciativa, la empatía, el optimismo, la creatividad o el estilo emocional. En el mercado de la formación directiva no se encuentran generalmente acciones formativas destinadas al desarrollo de estas compe- tencias, sino que se trabajan como parte del desarrollo de lo que se ha considerado habilidades directivas o ejecutivas.

3. Las competencias emocionales ejecutivas son competencias muy específicas adaptadas al mundo empresarial (aunque aplicable a las organizaciones complejas en general, por ejemplo las que no tienen ánimo de lucro). Su correcto dominio requiere de la ayuda directa de las competencias básicas y del apoyo indirecto de las competencias subyacentes. Son competencias asociadas a las habilidades directivas más tradicionales y son más fácilmente entrenables a través de acciones formativas específicas. Para identificarlas realizamos una búsqueda exhaustiva de acciones formativas destinadas a desarrollar habilidades directivas, siendo las más frecuentes acciones ofrecidas en el mercado de la formación directiva las siguientes: negociación, trabajo en equipo, gestión del estrés, escucha activa, comunicación efectiva, delegación, gestión de la incertidumbre, asertividad y gestión del cambio.

En definitiva, resulta muy difícil que un directivo líder pueda dirigir adecuadamente un equipo, consiguiendo su compromiso y coordinación, si no ejerce una serie de competencias emocionales básicas como la empatía (que permite una conexión emocional), la flexibilidad (imprescindible cuando los equipos son multiculturales) y la iniciativa (para plantear retos, establecer calendarios, proponer soluciones). Y una competencia emocional básica como la empatía, no puede ejercerse si a su vez no tiene una serie de competencias emocionales subyacentes como la autoestima y la adecuada percepción de las emociones de los otros. 
Jerarquización de competencias emocionales a través del modelo de la pirámide... De José Belzunce, M.; Danvila del Valle, I.; Martínez-López, F. y Sastre Castillo, M.

Esta idea clave se refleja en el modelo gráfico que denominamos pirámide invertida de las competencias emocionales (PICE) (Figura 2).

En la parte superior de la pirámide se encuentran las competencias ejecutivas: un número relativamente elevado de competencias (9), con un alto nivel de visibilidad. En la parte inferior, se encuentran las 4 competencias subyacentes, poco visibles en el actuar cotidiano, pero que influyen en todo el resto de competencias emocionales. En el nivel intermedio, hay 6 competencias emocionales básicas que son influidas por las subyacentes y a la vez influyen sobre las ejecutivas.

Las relaciones verticales describen conexiones entre competencias de distinto nivel, esto es: las competencias subyacentes con las básicas, y las básicas con las ejecutivas. Este análisis ha sido realizado teniendo en cuenta:
- La naturaleza de su concepto.

- Las investigaciones académicas que ponen de manifiesto sus relaciones.

- La necesidad de limitar y priorizar el número de conexiones entre las competencias.

Es cierto que todas están relacionadas entre sí, pero por razones metodológicas y prácticas, es conveniente limitar el número de asociaciones a un máximo, según la jerarquía en que se encuentren. En caso contrario, sería muy difícil (de cara a su aplicación práctica) su gestión eficiente y la extracción de conclusiones. Así, se ha realizado la asociación en función de las siguientes reglas:

- Las competencias del nivel más bajo (4 competencias subyacentes) se pueden asociar, como máximo, a la mitad +1 de las competencias del nivel inmediatamente superior $(6 \mathrm{com}$ petencias básicas).

Figura 2

\section{La pirámide invertida de las competencias emocionales}

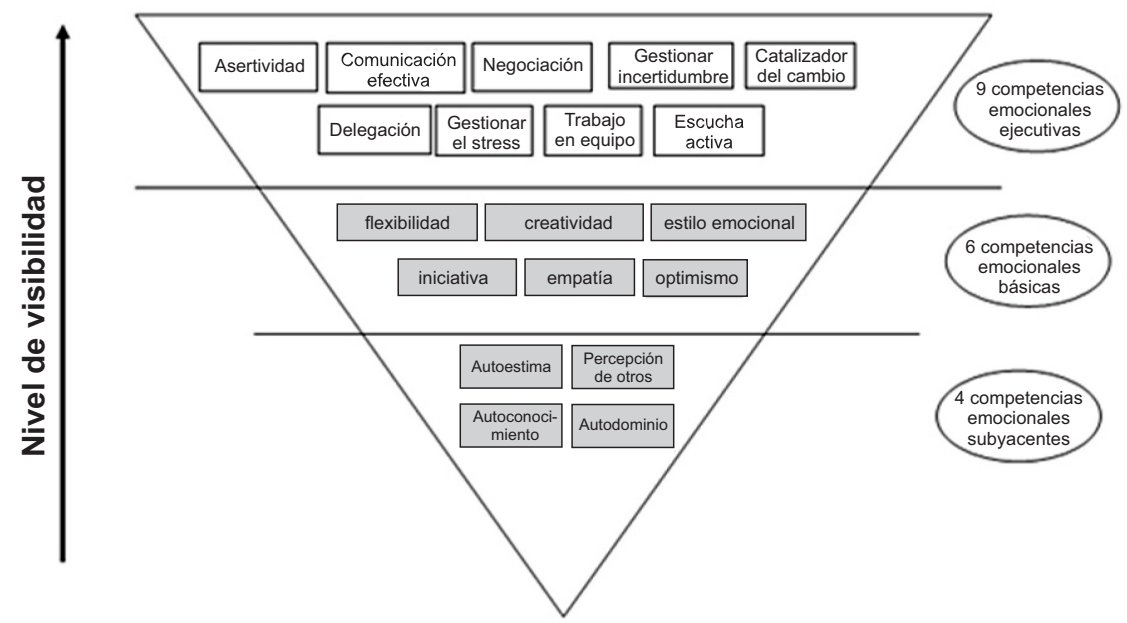

Fuente: Elaboración propia. 
- Las competencias del nivel básico, igualmente, se asocian, como máximo, con la mitad + 1 de las competencias del nivel superior (5 competencias ejecutivas).

Por tanto, cada competencia subyacente sólo puede asociarse a un máximo de 4 competencias básicas, y cada competencia básica puede asociarse a un máximo de 5 competencias ejecutivas. Esta forma de conectar las competencias entre sí, de forma jerárquica, va a poner de manifiesto que:

- Hay algunas competencias emocionales ejecutivas que tienen un alto nivel de conexión con un número elevado de competencias del nivel inferior (por ejemplo, la competencia de "negociación"). A su vez, hay otras com- petencias que tienen un grado de conexión mucho más bajo (por ejemplo, "la delegación" o "la asertividad").

- De forma similar, hay competencias básicas que tienen una densidad de conexiones con competencias subyacentes grandes (por ejemplo la empatía) frente a otras más bajas (por ejemplo la creatividad).

En definitiva, esta diferente densidad de conexiones es una medida del grado de dificultad que supone dominar una competencia. En general, se puede concluir que a mayor densidad de conexiones, más difícil resultará dominar una competencia y viceversa. El mapa de conexiones jerárquicas (verticales) mostrado en la Figura 3 ilustra las relaciones comentadas.

Figura 3

Mapa de conexiones jerárquicas entre competencias emocionales

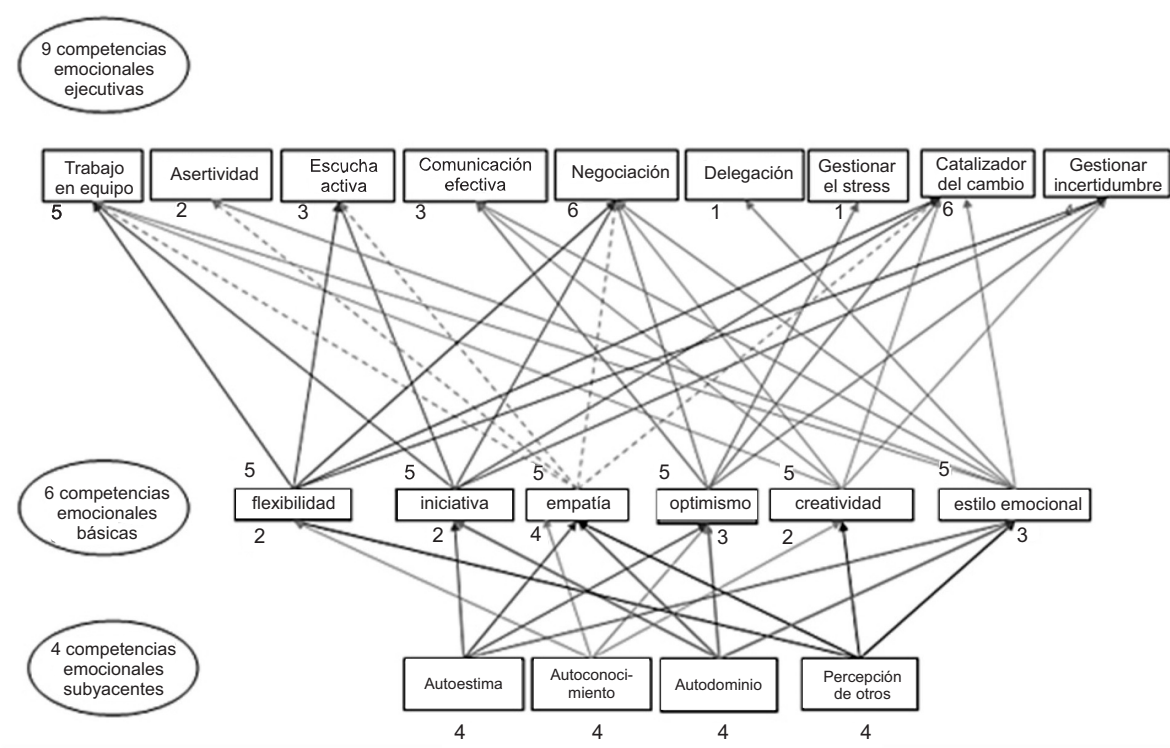

Fuente: Elaboración propia. 
Jerarquización de competencias emocionales a través del modelo de la pirámide... De José Belzunce, M.; Danvila del Valle, I.; Martínez-López, F. y Sastre Castillo, M.

Este procedimiento metodológico basado en conectar o relacionar las competencias emocionales entre sí tiene su base conceptual en los trabajos de McClelland (1973), quien consideró que el éxito en el trabajo se puede alcanzar con distintas combinaciones de competencias, que denomina algoritmos o conjuntos de competencias. A fin de demostrar el dominio en una competencia, hay que dominar otras competencias.

Por otra parte las competencias emocionales ejecutivas entran todas a formar parte de lo que tradicionalmente se denomina como habilidades directivas. Por tanto, lo que se pone de manifiesto es, exclusivamente, el valor añadido que aporta la inteligencia emocional, para completar y enriquecer estas habilidades directivas. Existen habilidades directivas que, por su mínimo componente emocional, son consideradas mayoritariamente como competencias técnicas. Por ello, no han sido incluidas dentro de la clasificación de competencias emocionales ejecutivas utilizada en el trabajo. En concreto, se utilizó la estructura de nueve competencias emocionales ejecutivas: trabajo en equipo, asertividad, escucha activa, comunicación efectiva, negociación, delegación, gestionar el stress, catalizador del cambio y gestionar de la incertidumbre.

Goleman (2005) sigue otro criterio, al no tener en cuenta el nivel o grado de influencia de la parte emocional frente a la cognitiva, y por tanto es menos restrictivo. Las competencias directivas que no se incluyen como competencias emocionales ejecutivas son: visión estratégica, gestión de riesgos, visión organizativa, gestión de proyectos, orientación al cliente, calidad total, excelencia, técnicas de toma de deci- siones, presentaciones efectivas, networking, control por excepción y, feedback.

Las competencias emocionales ejecutivas son aquéllas más visibles en la actuación de los líderes, y los más frecuentemente sujetos a crítica o admiración. También son las que se entrenan más habitualmente (mediante cursos, seminarios, jornadas o masters) y sobre los que se escriben decenas de libros al año.

\section{Estudio exploratorio de las competencias emocionales a partir de un panel de expertos}

En el presente trabajo, como principal objetivo de la investigación, además de proponer un modelo de niveles de competencias, se ha tratado de dar un paso adelante para su jerarquización, a través de la ponderación de las distintas competencias según la importancia que les conceden los responsables de las decisiones empresariales, para lo cual se ha propuesto un estudio basado en la opinión de expertos.

Para realizar este estudio que pretendía generar como resultado un mapa conceptual de competencias emocionales, se creó un panel Delphi, donde los participantes debían priorizar, entre la lista de competencias emocionales, cuáles de ellas consideraban esenciales.

Para ello se utilizó el sistema de encuestas vía Internet de www.surveymonkey.com, con una presentación previa de los objetivos e instrucciones para cumplimentar las encuestas, y una clarificación de los conceptos relativos a la IE y a las competencias emocionales.

A los encuestados se les presentó solamente las competencias ejecutivas (9) 
y las básicas (6), no las subyacentes (4), pues en un test previo se detectó que al resultar éstas demasiado generales daban lugar a confusión y malinterpretación.

Para asegurar una adecuada representatividad de la muestra en el Panel, los investigadores se dirigieron a todas las empresas del IBEX-35, y se obtuvo respuesta de 16 de ellas, recogidas en la Tabla 1, que representan el $35 \%$ de la facturación de las 100 empresas españolas mayores en tamaño de ventas y empleados). La solicitud de responder a la encuesta iba acompañada de una petición para poder realizar una entrevista en profundidad.

La obtención de los resultados de la encuesta ha sido laboriosa. Ninguna de estas empresas responde a encuestas enviadas por correo. Algunas tienen como política de empresa (por ejemplo Gamesa) no responder nunca a cuestionarios de trabajos de investigación ni encuestas de ningún tipo. Otras han declinado contestar por considerar que las preguntas tenían un carácter "sensible". Por ello, la mayoría de las respuestas se han obtenido recurriendo a contactos personales y profesionales.

Los directivos que participaron en el Panel se distribuyeron por niveles de responsabilidad según la siguiente proporción: 2 Vicepresidentes, 2 Directores Generales, 4 Directores de áreas staff (Recursos Humanos, Desarrollo de $\mathrm{Ne}$ gocio) y, 8 Directores Funcionales.

Los resultados se obtuvieron a través de un Delphi con dos rondas de respuestas, a través de la encuesta y entrevistas en profundidad, con las que se procedió a analizar y filtrar los datos, y a cuantificarlos dentro del modelo de la pirámide invertida de las competencias emocionales (PICE).

Después de la primera ronda de puntuación de las competencias se sintetizaron los resultados con las puntuaciones directas obtenidas por cada competencia en cada categoría, y se enviaron nuevamente a los participantes para que

Tabla 1

Empresas del IBEX-35 que forman parte de la muestra de estudio

\begin{tabular}{llr}
\hline \multicolumn{1}{c}{$\begin{array}{c}\text { EMPRESAS DEL IBEX-35 QUE HAN } \\
\text { RESPONDIDO A LA ENCUESTA }\end{array}$} & $\begin{array}{c}\text { Ventas } 2007 \\
\text { (miles } \boldsymbol{\epsilon} \text { ) }\end{array}$ \\
\hline 1 TELEFÓNICA & 57.807 .000 \\
2 REPSOL YPF & 54.692 .000 \\
3 IBERDROLA & 24.850 .872 \\
4 ENDESA & 20.774 .000 \\
5 B SANTANDER & 14.882 .000 \\
6 FERROVIAL & 12.370 .000 \\
7 GAS NATURAL & 10.221 .000 \\
8 BBVA & 9.769 .000 \\
9 FCC CONSTRUCCIÓN & 6.922 .000 \\
10 & ACCIONA S.A. & 6.818 .215 \\
11 ABERTIS & 3.620 .136 \\
12 B POPULAR & 2.339 .000 \\
13 INDRA SISTEMAS & 1.413 .331 \\
14 TÉCNICAS REUNIDAS & 1.247 .176 \\
15 RED ELÉCTRICA DE ESPAÑA & 1.031 .000 \\
16 BME & 383.000 \\
& TOTAL FACTURACIÓN & 229.139 .730 \\
\hline
\end{tabular}

Fuente: Elaboración propia sobre la base de las memorias publicadas por las empresas del IBEX-35. 
Jerarquización de competencias emocionales a través del modelo de la pirámide... De José Belzunce, M.; Danvila del Valle, I.; Martínez-López, F. y Sastre Castillo, M.

volvieran a priorizar las competencias, según su criterio, pudiendo reconsiderar su valoración inicial una vez conocidas las opiniones del resto de los participantes en el panel.

A estos efectos, se contó con unas valoraciones de partida para las competencias emocionales, en función de su tipología, y según cómo hubieran sido priorizados en primer, segundo o tercer lugar; el resto se valoró como cero.

El motivo por el cual se propuso estas puntuaciones, y no otras, se basó en que:

- Las competencias ejecutivas por ser más visibles y fácilmente identificables, reciben mayor puntuación que las menos visibles: las básicas (la básica más priorizada puntúa igual que la ejecutiva menos valorada) y las subyacentes (la subyacente más priorizada puntúa igual que la media de las ejecutivas valoradas).

- Las diferencias de puntuación entre las competencias ejecutivas son de 1 punto cada una, y entre las básicas y subyacentes, de medio punto.

Por otro lado, se obtuvo de forma indirecta la puntuación de las competencias emocionales subyacentes, de la siguiente manera:

- Toda competencia básica tiene asignadas unas conexiones verticales con competencias subyacentes. Si una competencia básica queda en primer lugar, le corresponden 2 puntos. $Y$ toda competencia subyacente con la que esté conectada (pueden ser más de una), recibirá 1,5 puntos.

- A su vez, toda competencia ejecutiva tiene conexiones verticales con com- petencias básicas. Así, una competencia ejecutiva que haya salido en segundo lugar, implica 1,5 puntos para las competencias básicas con las que tenga conexión vertical (pueden ser varias).

- Las puntuaciones se agrupan, tanto en las básicas (las que recibe de forma directa, a las que se le suman las que recibe de forma indirecta), como las subyacentes (que solo reciben las derivadas de las básicas). Solo las competencias ejecutivas tienen puntuaciones exclusivamente directas.

Posteriormente había que proceder a reflejar los resultados de las puntuaciones en el gráfico de la PICE. De la encuesta saldrían 3 competencias ejecutivas prioritarias y 3 básicas (las subyacentes, como se ha señalado, se calculan de forma indirecta).

El siguiente paso consistió en identificar los clusters de competencias verticales de las competencias priorizadas.

Se dispuso de varios mapas de relaciones verticales entre las competencias emocionales, y se trató de calcular las puntuaciones en función de los resultados de las encuestas, y de los mapas de conexiones entre competencias.

El resultado fue un mapa primario y uno secundario de competencias de la empresa, aquellas competencias que no obtenían puntuación alguna podían ser eliminadas del mapa de competencias para mayor facilidad y visibilidad interpretativa.

Para ello se procedió a colocar las puntuaciones sobre el mapa de competencias emocionales, descartando las competencias sin valor y manteniendo sólo las 3 competencias de mayor puntuación dentro 
de cada categoría (ejecutivas, subyacentes y básicas). Para ello, en la segunda ronda del panel Delphi se observó un incremento en la curtosis en torno a los valores más puntuados, con lo que las competencias eliminadas tenían en todos los casos unas puntuaciones claramente inferiores a las tres elegidas dentro de cada categoría de competencias.

En consecuencia, como resultado del proceso, se obtuvo el mapa general de competencias, en el que puede ser identificado el cluster vertical de primera prioridad, conectando verticalmente desde arriba hacia abajo, la competencia con mayor puntuación dentro de cada categoría, descartando todas las competencias no afectadas. Con ello se podía identificar el cluster vertical de segunda prioridad, conectando verticalmente desde arriba hacia abajo, la competencia con la segunda mayor puntuación dentro de cada categoría y descartando igualmente todas las competencias no afectadas (Figura 4).

Como se puede observar en la Figura 4, en las competencias ejecutivas, hay una puntuación única y directa, después de las dos rondas del Delphi, siendo las priorizadas, en este orden: escucha activa, comunicación eficaz y catalizador del cambio.

Sin embargo, en cuanto a las competencias básicas, hay puntuaciones obtenidas de forma directa (flexibilidad, iniciativa y optimismo), y otras de forma indirecta (puntuaciones obtenidas por su conexión con las competencias ejecutivas). De la combinación de todas, resultó que las competencias básicas más importantes fueron la iniciativa (6 puntos), la flexibilidad (5 puntos) y el optimismo (3,5 puntos), seguidas muy de cerca por la empatía (3 puntos).

En el tercer nivel, se tienen las puntuaciones de las competencias subyacen-

Figura 4

Mapa general de competencias emocionales según los expertos del Panel

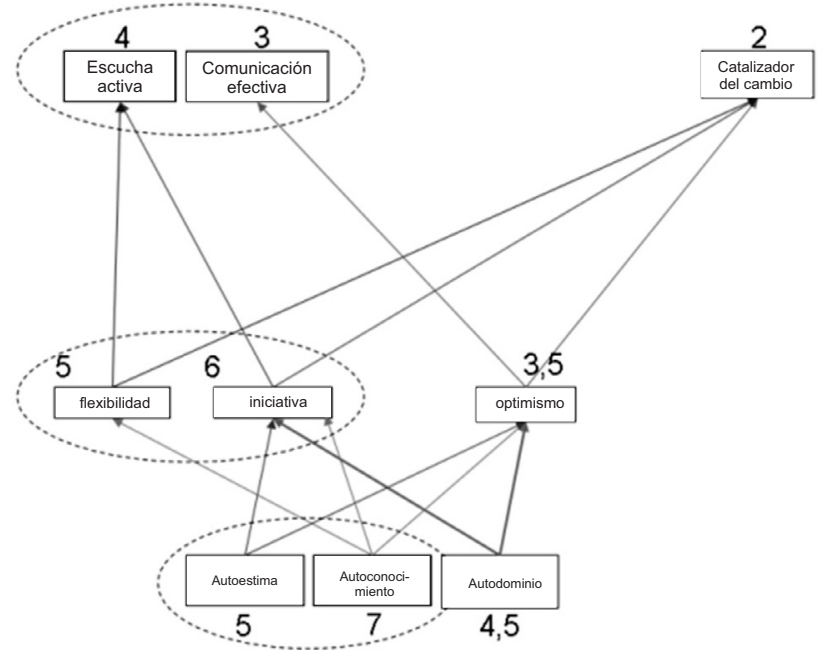

Fuente: Elaboración propia. 
Jerarquización de competencias emocionales a través del modelo de la pirámide... De José Belzunce, M.; Danvila del Valle, I.; Martínez-López, F. y Sastre Castillo, M.

tes, todas indirectas, derivadas de las conexiones verticales con las competencias básicas. Las que resultaron priorizadas fueron el autoconocimiento (7 puntos), la autoestima (5 puntos) y el autodominio (4,5 puntos).

Aplicando las competencias resultantes y sus puntos, sobre un mapa de competencias (que debería ser representativo de las empresas del lbex-35), se obtuvo el mapa representado en la Figura 4.

En la Figura 4 se reflejan las puntuaciones relativas a cada competencia emocional, habiendo eliminado de la misma las competencias sin puntuación, para favorecer el análisis. Dentro del mismo se han sombreado más oscuras las competencias clave dentro de cada categoría: la escucha activa, entre las ejecutivas; la iniciativa, entre las básicas; y el autoconocimiento, entre las subyacentes.
Se han reflejado, en tono intermedio, las competencias clave en segundo lugar, dentro de cada categoría de competencias: la comunicación efectiva, entre las ejecutivas; la flexibilidad, entre las básicas; y la autoestima, entre las subyacentes.

Se ha querido incluir también, porque la puntuación es muy similar, y parece relevante, un tercer grupo formado por el cambio, el optimismo, y el autodominio, sombreadas en tono más claro.

Este mapa general permite centrar los esfuerzos en 9 competencias emocionales de las 19 competencias del modelo, pero que realmente son 6 ya que las competencias subyacentes se forman de manera indirecta, e incluso los podemos reducir a 4: 2 de competencias ejecutivas y 2 de competencias básicas.

El cluster vertical (Figura 5) principal conecta las competencias emociona-

Figura 5
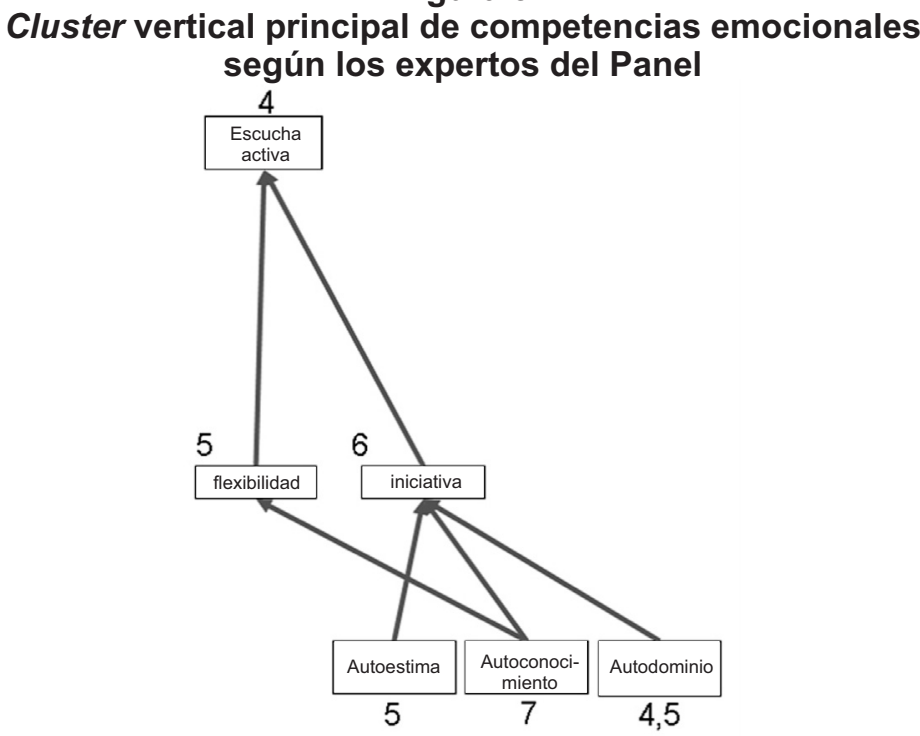

Fuente: Elaboración propia. 
les más demandadas dentro de cada categoría. En las figuras se señalan en oscuro tanto la competencia más votada de cada categoría, como las relaciones verticales que parten de la competencia ejecutiva, hasta llegar a las competencias subyacentes.

Como se observa en la Figura 5, de las competencias analizadas, la escucha activa es la más demandada por la empresa.

El cluster vertical secundario (Figura 6) conecta las competencias emocionales más demandadas en segundo lugar, dentro de cada categoría, señalando las relaciones verticales que parten de la comunicación efectiva, hasta llegar a las competencias subyacentes a las que está conectada a través de la competencia del optimismo.
En este caso, no había conexiones directas entre la comunicación efectiva y las 2 principales competencias básicas, en cuyo caso hubo que acudir a la tercera más votada, de ahí la utilidad del mapa general de competencias.

\section{Conclusiones}

El análisis de la literatura permitió establecer una clasificación de las competencias emocionales a partir de su naturaleza en subyacentes, básicas y ejecutivas, siendo esta propuesta la base de nuestro modelo de la pirámide invertida PICE. Esto permitiría dar respuesta al objetivo principal de nuestra investigación, como era el de relacionar y jerarquizar las competencias recogidas en dicho modelo. La validación vino a cargo de un panel

Figura 6

\section{Cluster vertical secundario de competencias emocionales} según los expertos del Panel

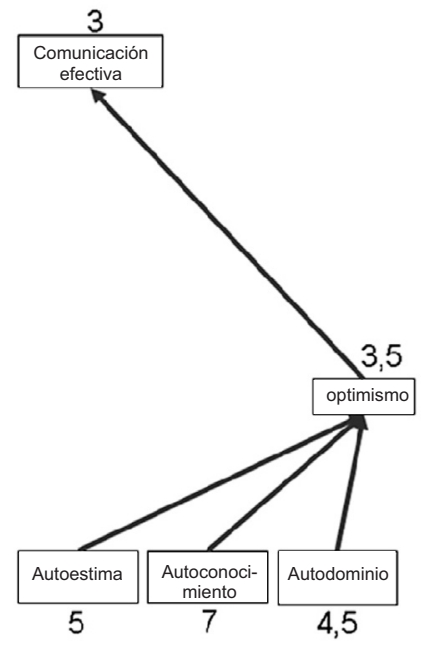

Fuente: Elaboración propia. 
Jerarquización de competencias emocionales a través del modelo de la pirámide... De José Belzunce, M.; Danvila del Valle, I.; Martínez-López, F. y Sastre Castillo, M.

Delphi constituido por directivos de las más relevantes empresas españolas que cotizan en Bolsa, a partir del cual se pudo extraer un conjunto de conclusiones extrapolables a otras empresas.

Entre las competencias emocionales ejecutivas, se encontró que la escucha activa era la más importante según el consenso de los expertos, seguida de la comunicación efectiva y de ser catalizador del cambio, estando las dos primeras relacionadas entre sí. Profundizando en el proceso metodológico seguido para obtener ese resultado, se encontró que se podía inferir de las entrevistas en profundidad realizadas, que existía a menudo un déficit de fluidez de comunicación interna, con causas diversas (departamentos muy jerarquizados verticalmente, escasa cooperación interdepartamental, dificultad de orientación común hacia las mismas metas o la existencia de conflictos de objetivos entre unidades organizativas) o en ocasiones, actitudes que denotaban falta de flexibilidad en las posturas y falta de iniciativa para resolverlas.

Las empresas, en la situación actual, parecen necesitar de unos directivos con alta iniciativa y una importante actitud hacia la escucha activa, donde puede haber oportunidades no aprovechadas tanto hacia el mercado (escucha al cliente) como internas (posibles reducciones de costos, reorganizaciones internas que den mayor eficiencia, entre otras).

Aparte de estas reflexiones que, como consecuencia práctica, pueden servir como punto de partida de un diagnóstico de competencias emocionales a debatir con el equipo directivo, el modelo PICE permite operativizar el cambio necesario, en el siguiente sentido: se pue- den crear programas de acción para aumentar y desarrollar las competencias emocionales críticas.

Los mapas de competencias y los clusters sirven para dar un enfoque global. Así, un programa de desarrollo interno de la competencia escucha activa, debería recoger como parte fundamental del mismo, el trabajo con las competencias que la soportan, la flexibilidad y la iniciativa. De forma análoga, la competencia ejecutiva de comunicación efectiva no debe ser abordada de manera aislada, sino que debería abordarse, como una parte sustancial del programa, el desarrollo de la competencia optimismo.

De las entrevistas realizadas, se ha observado que las empresas, posiblemente como consecuencia de la actual crisis, están pasando por una etapa de cierto desánimo y, en ocasiones, desconcierto, sin una visión clara compartida, y con un bajo nivel de optimismo en las relaciones emocionales individuales y grupales. De aquí se pueden definir planes de acción más específicos, aplicables al entrenamiento de los directivos, trabajando la competencia optimismo como parte preliminar del desarrollo de la comunicación efectiva.

En síntesis, la utilidad del modelo propuesto, estriba en la posibilidad de aportar pistas sobre cómo desarrollar las competencias que permitan resolver problemas existentes en una empresa y aprovechar posibles oportunidades de mejora, visualizando las relaciones entre las competencias donde la empresa tiene puntos fuertes o bien debilidades.

Un adecuado autodiagnóstico es lo que permitiría encontrar el déficit de habilidades directivas en una empresa, a par- 
tir de las cuales, con base en el modelo propuesto en este trabajo, diseñar los programas adecuados para su desarroIlo, conociendo las competencias emocionales básicas que debieran ser trabajadas.

Sin embargo se trata de una aproximación, de un estudio exploratorio, pero tanto las ponderaciones de las competencias en sus tres niveles como las relaciones entre ellas deberán ser contrastadas en futuros trabajos para poder delimitar el alcance de la validez del modelo propuesto.

\section{Referencias bibliográficas}

Bar-on, Reuven (1997). Bar-On Emotional Quotient Inventory: Technical manual, Multi-Health Systems, Toronto, Canadá.

Bar-on, Reuven (2000). Emotional and social intelligence: insights from the emotional quotient inventory (EQ-I), en: Baron, Reuven and Parker, James. The Handbook of Emotional Intelligence, Jossey-Bass, San Francisco, pp. 363-388.

Boyatzis, Richard (1982). The Competent Manager: A Model for Effective Performance, Wiley \& Sons, New York, Estados Unidos.

Boyatzis, Richard (2006). Intentional change theory from a complexity perspective, Journal of Management Development, vol. 25, $n^{\circ} 7$, pp. 607-623.

Boyatzis, Richard y Saatcioglu, Argun (2008). A 20-year view of trying to develop emotional, social and cognitive intelligence competencies in graduate management education, Journal of Management Development, vol. $27, n^{\circ}$ 1, pp. 92-108.
Boyatzis, Richard y Sala, Fabio (2004). Assessing emotional intelligence competencies, en: Geher, Glenn. The Measurement of Emotional Intelligence, Novas Science Publishers, Hauppauge, Nueva York.

Cherniss, Cary (2001). Emotional intelligence and organizational effectiveness, en: Cherniss, Cary y Coleman, Daniel. The Emotionally Intelligent Workplace, Jossey-Bass, San Francisco.

Danvila del Valle, Ignacio y Sastre Castillo, Miguel Ángel (2010). Inteligencia Emocional: una revisión del concepto y líneas de investigación, Cuadernos de Estudios Empresariales, vol. 20, pp. 103-127.

Dulewicz, Victor y Higgs, Malcolm (1998). Can emotional intelligence be measured and developed?, Leadership \& Organization Development Journal, vol. $20, n^{\circ} 5$, pp. 242-252.

Dulewicz, Victor y Higgs, Malcolm (2000). Emotional intelligence: a review and evaluation study, Journal of Managerial Psychology, vol. $15 n^{\circ} 4$, pp. 341-372.

Emmerling, Robert y Goleman, Daniel (2003). Emotional Intelligence: Issues and Common Misunderstandings. Consultado el 07 de noviembre de 2012 en: http://www.eiconsortium.org/reprints/ei_issues_and_common_misunderstandings.html.

Fernández-Berrocal, Pablo y Extremera, Natalio (2006). Métodos de evaluación de la inteligencia emocional en el aula, en Gómez, Ángel, Gaviria, Elena y Fernández, Itziar Psicología social, Sanz \& Torres, Madrid.

Gardner, Howard (1983). Frames of Mind: The Theory of Multiple Intelligences, Basic Books, New York.

Gardner, Howard (1993). Multiple intelligences: The theory in practice, Basic Books, New York. 
Jerarquización de competencias emocionales a través del modelo de la pirámide...

De José Belzunce, M.; Danvila del Valle, I.; Martínez-López, F. y Sastre Castillo, M.

Gardner, Lisa y Stough, Con (2002). Examining the relationship between leadership and emotional intelligence in senior level managers, Leadership \& Organization Development Journal, vol. $23 n^{\circ} 2$, pp. 68-78.

George, Jennifer (1995). Leader positive mood and group performance: the case of customer service, Journal of Applied Social Psychology, $n^{\circ} 25$, pp. 778-794.

George, Jennifer (2000). Leader positive mood and group performance: the case of customer service, Human Relations, vol. 53, n 8, pp. 1027-55.

George, Jennifer y Bettenhausen, Kenneth (1990). Understanding prosocial behavior, sales performance, and turnover: a group level analysis in a service context. Journal of Applied Psychology, $n^{\circ} 75$, pp. 698-709.

Goleman, Daniel (1995). Emotional Intelligence, Bantam Books, New York.

Goleman, Daniel (1998). La práctica de la inteligencia emocional, Kairós, Barcelona.

Goleman, Daniel (2001). Emotional Intelligence: Issues in Paradigm Building, en: Cherniss, Cary y Coleman, Daniel The Emotionally Intelligent Workplace, Jossey-Bass, San Francisco.

Goleman, Daniel; Boyatzis, Richard y McKee, Annie (2002a). The New leaders. Harvard Business School Press, Boston.

Goleman, Daniel; Boyatzis, Richard y McKee, Annie (2002b). El líder resonante crea más, Plaza y Janés, Barcelona.

Goleman, Daniel; Boyatzis, Richard y McKee, Annie (2004). Primal Leadership. Learning to lead with emotional intelligence, Harvard Business School Press, Boston.

Higgs, Malcolm y Rowland, Deborah (2002). Does it need emotional intelligence to lead change?, Journal of General Management, $n^{\circ} 27$, pp. 62-76.
Howard, Ann y Bray, Douglas (1998). Managerial Lives in Transition: Advancing Age and Changing Times. Guilford Press, New York.

Jamali, Dima; Sidani, Yusuf y Abu-Zaki, Dana Mohammed (2008). Emotional intelligence and management development implications. Insights from the Lebanese context, Journal of Management Development, vol. $27 n^{\circ} .3$, pp. 348-360.

Koman, Elizabeth Stubbs y Wolff, Steven B. (2008). Emotional intelligence competencies in the team and team leader: $A$ multi-level examination of the impact of emotional intelligence on team performance, Journal of Management Development, vol. 27, n 1, pp. 55-75.

Koontz, Harold y O’Donnell, Cyril (1964). Principles of Management: An Analysis of Managerial Functions, McGraw Hill, New York.

Leuner, Benedetta (1966). Emotional intelligence and emancipation, Praxis der Kinderpsychologie und Kinderpsychiatrie, vol. 15, pp. 193-203.

Mandell, Barbara y Pherwani, Shilpa (2003). Relationship between emotional intelligence and transformational leadership style: a gender comparison, Journal of Business and Psychology, vol. 17, $n^{\circ} 3$, pp. 387-404.

Mayer, John y Salovey, Peter (1997). What is emotional intelligence?, en Salovey, Peter y Sluyter, David Emotional development and emotional intelligence: Educational implications, Basic Books, New York, pp. 3-31.

Mayer, John; Caruso, David y Salovey, Peter (1999). Emotional Intelligence: meets traditional standards for an intelligence, Intelligence, vol. 27, $\mathrm{n}^{\circ} 4$, pp. 267-298.

McClelland, David (1973) Testing for competence rather than intelligence, American Psychologist, vol. 28, $\mathrm{n}^{\circ} 1$, pp. 1-40. 
Nikolaou, Ioannis y Tsaousis, Ioannis (2002). Emotional intelligence and occupational stress, The International Journal of Organizational Analysis, vol. 10, $n^{\circ} 4$, pp. 327-342.

Payne, Wayne (1986). A study of emotion: Developing emotional intelligence: Selfintegration; relating to fear, pain and desire. Dissertation Abstracts International, vol. 47, pp. 203 (UMI No. AAC 8605928).

Prati, Melati; Douglas, Ceasar; Ferris, Gerald; Ammeter, Anthony y Buckley, Ronald (2003). Emotional intelligence, leadership effectiveness and team outcomes, The International Journal of Organizational Analysis, vol. 11, $\mathrm{n}^{\circ}$ 1, pp. 21-40.
Salovey, Peter y Mayer, John (1990). Emotional intelligence, Imagination, Cognition and Personality, vol. 9, $n^{\circ} 3, p p$. 185-211.

Schmidt, Frank y Hunter, John (1998). The validity and utility of selection methods in personnel psychology: Practical and theoretical implications of 85 years of research findings, Psychological Bulletin, $n^{\circ} 124$, pp. 262-274.

Van Ghent, Dorothy (1961). The English novel: Form and function, Harper \& Row, New York.

Weisinger, Hendrie (1998). Emotional Intelligence at Work, Jossey-Bass, San Francisco. 